\title{
GROUP ALGEBRAS WHOSE SIMPLE MODULES ARE INJECTIVE
}

\author{
BY \\ DANIEL R. FARKAS(1) AND ROBERT L. SNIDER
}

\begin{abstract}
Let $F$ be either a field of char 0 with all roots of unity or a field of char $p>0$. Let $G$ be a countable group. Then all simple $F[G]$-modules are injective if and only if $G$ is locally finite with no elements of order char $F$ and $G$ has an abelian subgroup of finite index.
\end{abstract}

The condition that all simple modules over a ring be injective first appeared in a theorem due to Kaplansky: a commutative ring satisfies the condition if and only if it is von Neumann regular. Several people have studied the property for noncommutative rings, a recent example being [3]. The authors of that paper suggest the problem of characterizing group algebras with this condition. In this paper we make substantial progress by offering

Theorem 3. Let $F$ be either a field of char 0 with all roots of unity or a field of char $p>0$. Let $G$ be a countable group. Then all simple $F[G]$-modules are injective if and only if $G$ is locally finite with no elements of order char $F$ and $G$ has an abelian subgroup of finite index.

The proof is divided into three parts. In $\S 1$, we show that $F$ is injective as an $F[G]$-module if and only if $G$ is locally finite with no elements of order char $F$. In the second and crucial section, we show that for a certain class of rings ("locally Wedderburn algebras") the condition that all simple modules are injective is equivalent to the property that all simple modules are finite dimensional over their commuting rings. In $\S 3$, we prove the main theorem by showing that if all simple modules are finite dimensional over their commuting rings then $G$ is abelianby-finite.

We would like to thank D.S. Passman for suggestions that shortened and improved our work.

1. Results of Villamayor. Most of this section can be gleaned from several of Villamayor's papers. However, the statements and proofs that appear here are new.

The field $F$ is a right $F[G]$-module under the trivial action (i.e. if $k \in F$, then $k \cdot a=k(a) \varepsilon$ where $\varepsilon$ is the augmentation map). We characterize those $G$ with $F$ being $F[G]$-injective. By considering maps from ideals of $F[H]$ to $F$, the reader can casily prove

Received by the editors February 5, 1973.

AMS (MOS) subject classifications (1970). Primary 16A26, 20C05; Secondary 16A52, 16A64.

Key words and phrases. Injective simple module, group algebra, locally finite groups.

(1) The first author is on an NSF fellowship. His portion will constitute part of his doctoral thesis directed by I. N. Herstein at the University of Chicago. 
Lemma 1. If $H$ is a subgroup of $G$ and $F$ is $F[G]$-injective then $F$ is $F[H]$-injective.

Lemma 2. Let $G$ be a finite group. $F$ is $F[G]$-injective if and only if $|G| \neq 0$ in $F$.

Proof. If $|G| \neq 0$ on $F$, then by Maschke's theorem $F[G]$ is semisimple and the result follows.

Suppose ch $F=p>0$. By Lemma 1 the converse follows if we show that $F$ is not $F[G]$-injective for $G=\langle x\rangle$ cyclic of order $p$. Let $A$ be the augmentation ideal of $F[G]$ with $F$-basis $x-1, x^{2}-1, \ldots, x^{p-1}-1 . f: A \rightarrow F$ given by $\left(\sum \lambda_{i}\left(x^{i}-1\right)\right) f=\sum \lambda_{i} i$ is a well-defined $F[G]$-map. If $F$ is $F[G]$-injective $f$ can be extended to $F[G]$. That is, $\exists c \in F \ni 1=(x-1) f=c \cdot(x-1)=0$, a contradiction.

Theorem 1([3], [7]). $F$ is $F[G]$-injective if and only if $G$ is locally finite and the order of no element of $G$ vanishes in $F$.

Proof. Suppose $F$ is $F[G]$-injective and $g_{1}, \ldots, g_{n} \in G \ni\left\langle g_{1}, \ldots, g_{n}\right\rangle$ is infinite: Define $f: F[G] \rightarrow F[G]^{(n)}$ by $(r) f=\left(\left(g_{1}-1\right) r, \ldots,\left(g_{n}-1\right) r\right)$, an $F[G]-$ map. By $[4$, p. 105$] f$ is injective.

Complete

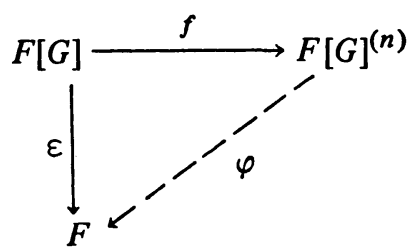

and set $\lambda_{i}=(0, \ldots, 0,1,0, \ldots, 0) \varphi$ with $i$ th coordinate $1.1=(1) \varepsilon=(1) f \varphi$ $=\left(g_{1}-1, \cdots, g_{n}-1\right) \varphi=\Sigma \lambda_{i} \cdot\left(g_{i}-1\right)=0$, a contradiction. Thus $G$ is locally finite; the other condition follows from the lemmas.

Conversely suppose $G$ is such a group, $0 \neq I$ is a right ideal of $F[G]$ and $0 \neq f: I \rightarrow F$ is an $F[G]$-map. 'If $u \in I \ni(u) f \neq 0$ let $G_{0}=\langle\operatorname{supp} u\rangle$, a finite group with $I \cap F\left[G_{0}\right] \neq 0$. By Lemma $2, f \mid I \cap F\left[G_{0}\right]$ can be extended to $F\left[G_{0}\right]$; i.e. $\exists \lambda \in F \ni a \in I \cap F\left[G_{0}\right] \Rightarrow(a) f=\lambda \cdot a=\lambda(a) \varepsilon$. Now let $b \in I$. There is a finite subgroup $G_{1}$ containing $G_{0}$ and supp $b$. As above $\exists \lambda^{\prime} \in F$ $\exists(b) f=\lambda^{\prime}(b) \varepsilon$ and $(u) f=\lambda^{\prime}(u) \varepsilon$. Then $\left(\lambda-\lambda^{\prime}\right)(u) \varepsilon=0$. But $(u) \varepsilon \neq 0$ since (u) $f \neq 0$. Therefore $\lambda=\lambda^{\prime}$. Thus $(b) f=\lambda \cdot b$ and $f$ can be extended.

2. Locally Wedderburn algebras. If all simple $F[G]$-modules are injective then Theorem 1 in conjunction with Maschke's theorem implies that $F[H]$ is a finite dimensional semisimple subalgebra for every finitely generated subgroup $H \subseteq G$. We isolate this situation.

Definition. Let $A$ be an algebra with 1 over $F$. $A$ is a locally Wedderburn algebra if every finite set of elements in $A$ is contained in a semisimple subalgebra finite dimensional over $F$.

Recall that, in general, if $V$ is an $A$-module and $T$ is a subset of $A$ then the annihilator $l(T)=\{v \in V \mid v t=0 \forall t \in T\}$. 
Lemma 3. Let $A$ be a locally Wedderburn algebra. Simple modules finite dimensional over their commuting rings are injective.

Proof. Let $V$ be a simple $A$-module finite dimensional over its commuting ring $C$. If $V$ is not injective there is a right ideal $I \subseteq A$ and an $A$-map $f: I \rightarrow V$ which cannot be lifted to $A$. Setting $D(B)=\{v \in V \mid v i=(i) f \forall i \in I \cap B\}$ for any subalgebra $B \subseteq A$, our assumption is $\bigcap_{B \in \zeta} D(B)=\varnothing$ where the intersection is taken over all finite dimensional semisimple subalgebras $B$. Since all modules over such an algebra are injective, $D(B) \neq \varnothing \forall B \in \zeta$.

Choose $B_{0} \in \zeta \ni d=\operatorname{dim}_{C} I\left(I \cap B_{0}\right)$ is minimal. By the empty intersection $\exists B_{1}$ $\in \zeta \ni D\left(B_{0}\right) \nsubseteq D\left(B_{1}\right)$. If $B_{2} \in \zeta$ contains a basis for $B_{0}$ and $B_{1}$ then $B_{0} \subseteq B_{2}$ implies $\varnothing \neq D\left(B_{2}\right) \subsetneq D\left(B_{0}\right)$. Thus if $\omega \in D\left(B_{2}\right), D\left(B_{2}\right)=\omega+l\left(I \cap B_{2}\right) \subsetneq \omega$ $+l\left(I \cap B_{0}\right)=D\left(B_{0}\right)$, contradicting the minimality of $d$.

The next four technical lemmas are needed to prove a converse to Lemma 3. The first is essentially due to D. S. Passman (private communication).

Lemma 4. Let $A$ be any countable union of $F$-algebras $A_{1} \subseteq A_{2} \subseteq A_{3} \subseteq \cdots$ (with the same 1) and let $V$ be an injective $A$-module of countable dimension over $F$. If $I$ is any right ideal of $A$ then the sequence $l\left(I \cap A_{1}\right) \supseteq l\left(I \cap A_{2}\right) \supseteq \cdots$ terminates.

Proof. Suppose that for some fixed $I$ the sequence does not terminate and write $L=\bigcap_{i} l\left(I \cap A_{i}\right)$. Let $W$ be the $F$-space of all countably infinite sequences in $F$. Then $\operatorname{dim}_{F} W$ is uncountable. We will construct a linear transformation $T: W$ $\rightarrow V / L$ which is one-to-one. Since $\operatorname{dim}_{F} V / L$ is countable this will yield the desired contradiction.

By renumbering we can assume that the annihilators strictly decrease. Choose $v_{i} \in l\left(I \cap A_{i}\right) \backslash l\left(I \cap A_{i+1}\right)$ for $i=1,2, \ldots$ Clearly $v_{1}, \ldots, v_{n}$ are $F$-linearly independent modulo $l\left(I \cap A_{n+1}\right)$. Fix $\omega=\left(\alpha_{1}, \alpha_{2}, \ldots\right) \in W$. The map $f_{n}: A_{n}$ $\rightarrow V$ given by $1 \rightarrow \sum_{i=1}^{n} \alpha_{i} v_{i}$ is an $A_{n}$-map. If $k \leq n$ and $a_{k} \in I \cap A_{k}$ then

$$
\left(a_{k}\right) f_{n}=\left(\sum_{i=1}^{n} \alpha_{i} v_{i}\right) a_{k}=\left(\sum_{i=1}^{k} \alpha_{i} v_{i}\right) a_{k}=\left(a_{k}\right) f_{k} .
$$

Thus there is an $A$-map $f: I \rightarrow V$ where $f\left|I \cap A_{n}=f_{n}\right| I \cap A_{n}$.

Since $V$ is injective $f$ lifts to $A$. Set $(1) f=\hat{\omega} \in V$. By construction $\hat{\omega}$ $-\sum_{i=1}^{n} \alpha_{i} v_{i} \in l\left(I \cap A_{n}\right)$. The correspondence $\omega \rightarrow \hat{\omega}$ induces a function $T: W$ $\rightarrow V / L$. Regarding $V / L$ as a subspace of $\prod_{n=1}^{\infty} V / l\left(I \cap A_{n}\right)$ we see

$$
\left(\alpha_{1}, \alpha_{2}, \ldots\right) \rightarrow\left(\overline{\alpha_{1} v_{1}}, \overline{\alpha_{1} v_{1}+\alpha_{2} v_{2}}, \ldots\right) .
$$

Thus $T$ is an $F$-linear map. $\omega T=0 \Rightarrow$ for all $n \geq 2, \sum_{i=1}^{n} \alpha_{i} v_{i} \in l\left(I \cap A_{n}\right) \Rightarrow$ for all $n \geq 2, \alpha_{1}, \ldots, \alpha_{m-1}=0$ by linear independence $\bmod A_{n}$. That is, $\omega=0$ and $T$ is one-to-one.

Lemma 5. Let $R \subseteq S$ be rings and $f: W \rightarrow V$ be an $R$-monomorphism from the 
simple R-module $W$ (with commuting ring $C$ ) to the simple $S$-module $V$ (with commuting ring $D$ ). If $\omega_{1}, \ldots, \omega_{n} \in W$ are independent over $C$ then $\omega_{1} f, \ldots, \omega_{n} f$ are independent over $D$.

Proof. If not, $\exists d_{i} \in D \ni \omega_{1} f=\sum_{i>1} d_{i}\left(\omega_{i} f\right)$. By density $\exists r \in R \ni \omega_{1} r$ $=\omega_{1}$ and $\omega_{i} r=0$ for $i>1$.

$$
\omega_{1} f=\left(\omega_{1} r\right) f=\left(\omega_{1} f\right) r=\sum d_{i}\left(\omega_{i} r\right) f=0 \Rightarrow \omega_{1}=0,
$$

a contradiction.

Lemma 6. Let $A$ be a countable dimensional locally Wedderburn algebra and let $\chi$ be a simple A.module infinite dimensional over its commuting ring $D$. Then there are finite dimensional semisimple subalgebras $A_{1} \subseteq A_{2} \subseteq A_{3} \subseteq \cdots$ and subspaces $V_{1}, V_{2}, V_{3}, \ldots$ of $\chi \ni$

(i) $A=\bigcup A_{i}$.

(ii) $V_{i}$ is a simple $A_{i}$-submodule with commuting ring $C_{i}$.

(iii) There are $A_{i}$-monomorphisms $\pi_{i}: V_{i} \rightarrow V_{i+1}$.

(iv) $\operatorname{dim}_{C_{i+1}} V_{i+1}>\operatorname{dim}_{C_{i}} V_{i}$.

Proof. Let $\left\{b_{1}, b_{2}, \ldots\right\}$ be a basis for $A$. We proceed by induction, proving that $b_{n} \in A_{n}$. Let $A_{1}$ be any finite dimensional semisimple subalgebra containing $b_{1}$ and let $V_{1}$ be any simple $A_{1}$-submodule of $\chi$. Assume $A_{m}$ and $V_{m}$ have been chosen. Let $u \in \mathcal{X}$ be $D$-independent of $V_{m}$ (such an element exists by a dimension argument). Since $V_{m}$ is finite dimensional, by density $\exists a \in A \ni V_{m} a$ $=0$ and $0 \neq u a \in V_{m}$. Let $A_{m+1} \subseteq A$ be a finite dimensional semisimple subalgebra containing $A_{m}, a$, and $b_{m+1} . \chi$ decomposes as some direct sum $\bigoplus_{\alpha} U_{\alpha}$ of simple $A_{m+1}$-submodules; let $\pi_{\alpha}: \chi \rightarrow U_{\alpha}$ be the canonical $A_{m+1}$-projection. Clearly $\exists \beta \ni(u a) \pi_{\beta} \neq 0$.

Set $V_{m+1}=U_{\beta}$. Then $\pi_{\beta}: V_{m} \rightarrow V_{m+1}$ is an $A_{m}$ monomorphism by the transitive action of $A_{m}$ on $V_{m}$. If $C_{m+1}$ is the commuting ring of $V_{m+1}$, then $u \pi_{\beta}$ and $V_{m} \pi_{\beta}$ are independent over $C_{m+1}$ since $\pi_{\beta}$ is an $A_{m+1}$-map and $a \in A_{m+1}$ annihilates $V_{m}$ and not $u$. Dimensions over the commuting rings go up by Lemma 5 .

Let $A$ be a countable dimensional locally Wedderburn algebra and $V$ be a simple $A$-module with commuting ring $D$. As in Lemma 6, we see $A=\cup A_{i}$ where each $A_{i}$ is semisimple. Since $A_{i}$ has only finitely many isomorphism classes of simple modules we know that $V=\bigoplus \sum_{j=1}^{t_{j}} V_{i j}$ where $V_{i j}$ is a homogeneous component for a suitable simple $A_{i}$-module. Furthermore each $V_{i j}$ is clearly a $D$ subspace.

Lemma 7. Let $A=\cup A_{i}$ be a countable dimensional locally Wedderburn algebra and let $V$ be a simple $A$-module infinite dimensional over its commuting ring $D$. Then for each $i$ there exists a simple $A_{i}$-module $U_{i}$ and $A_{i}$-monomorphism $f_{i}: U_{i} \rightarrow U_{i+1}$ such that the $U_{i}$ homogeneous component of $V$ (as an $A_{i}$-module) is infinite dimensional over $D$. 
Proof. For each $i, V=\bigoplus \sum_{j=1}^{t_{i}} V_{i j}$ and $\operatorname{dim}_{D} V=\infty$ whence for some $j$, $\operatorname{dim}_{D} V_{i j}=\infty$. Take as $U_{1}$ any simple $A_{1}$-submodule with infinite dimensional homogeneous component. Given $U_{n-1}$ then $U_{n-1} \subseteq \bigoplus \sum_{j=1}^{t_{n}} V_{n j}$ as $A_{n-1}$ modules. If $U_{n-1}$ is contained isomorphically in only the $D$ finite dimensional $V_{n j}$, then the infinite dimensional homogeneous component of $U_{n-1}$ must be included in this finite direct sum of finite dimensional subspaces, a contradiction. Hence $U_{n-1}$ is contained isomorphically in some infinite dimensional $V_{n j}$. Since $V_{n j}$ is a direct sum of simple $A_{n}$-modules, by looking at the projection maps we that $U_{n-1}$ is contained isomorphically in some $U_{n}$ with homogeneous component $V_{n j}$.

Theorem 2. Let $A$ be a countable dimensional locally Wedderburn algebra. All simple A-modules are injective if and only if all simple A-modules are finite dimensional over their commuting rings.

Proof. One direction has already been proved in Lemma 3. Thus we assume $A$ has a simple module infinite dimensional over its commuting ring and construct a simple module which is not injective. Following Lemma 6 we may assume that the infinite dimensional simple module is $v=U V_{i}$ with $V_{1} \subseteq V_{2} \subseteq \cdots$. If $C$ is the commuting ring of $V$ then $\operatorname{dim}_{C} V=\infty$ by Lemma 5 .

We first show that there exists an integer $N$ such that for all $n \geqslant N$, the homogeneous component of $V_{n}$ has finite $C$-dimension.

Suppose not.

Fix $0 \neq u \in V_{1}$. As a $C_{i}$-space $V_{i}=C_{i} u \oplus V_{1}^{\prime}$. Define $a_{i} \in A_{i}$ with action $\left(\begin{array}{l}0 \\ 0 \\ 0\end{array}\right)$ and set $I=a_{1} A+a_{2} A+\cdots$. We first show that $l_{v}(I)=C u$. Clearly $u a_{i}=0 \forall i$ and so $C u \subseteq l(I)$. If $v \in l(I)$ and if $u$ and $v$ are $C$-independent then $\exists b \in A \ni v \cdot b=v$ and $u \cdot b=0$. Choose $n \ni b \in A_{n}$ and $v \in V_{n} \cdot v a_{n}=0$ $\Rightarrow v$ and $u$ are $C_{n}$-dependent. Thus $u b=0 \Rightarrow v b=0 \Rightarrow v=0$, a contradiction.

By Lemma 4, there exists $j$ such that $\operatorname{dim}_{C} l\left(I \cap A_{j}\right)=1$. As an $A_{j}$-module the homogeneous component $T$ of $V$ corresponding to $V_{j}$ is the internal direct sum $\sum_{n=1}^{\infty} V_{j n}$ where $V_{j 1}=V_{j}$ and the subscript map $V_{j} \rightarrow V_{j n}$ is an $A_{j}$-isomorphism. $\left\{u_{n} \mid n=1,2, \ldots\right\} \subseteq l\left(I \cap A_{j}\right)$ since $u \in l(I)$. Thus there exists $c_{n}$ in $C$ such that $c_{n} u=u_{n}$. If $v$ is in $V_{j n}$ then there exists $x$ in $A_{j}$ such that $v=u_{n} x=c_{n} u x$ in $C V_{j}$. Thus $\operatorname{dim}_{C} T=\operatorname{dim}_{C_{j}} V_{j}<\infty$, a contradiction.

Pick $U_{n}$ as in Lemma 7. For $n \geqslant N, U_{n}$ is not isomorphic to $V_{n}$. By taking a subsequence we can further assume $U_{n} \subseteq V_{n+1}$.

Define $\varphi_{i}: U_{i} \oplus V_{i} \rightarrow U_{i+1} \oplus V_{i+1}$ by $(u, v) \varphi_{i}=\left((u) f_{i}, u+v\right)$, an $A_{i}$-monomorphism. The direct limit $W$ of this system is an $A$-module under the obvious action and the map $v \rightarrow(0, v)$ is an $A$-injection of $V$ into $W$. A typical nonzero element of $W$ can be represented by an ordered pair $(u, v)$ where $u \in U_{n}$ and $0 \neq v \in V_{n}$. Since $U_{n}$ and $V_{n}$ are nonisomorphic simple $A_{n}$-modules $\exists a \in A_{n}$ whose action on $V_{n}$ is the identity and whose action on $U_{n}$ is zero. That is, $(u, v) \cdot a=(0, v) \in V$ and so $W$ is a proper essential extension of $V$.

Corollary 1. Let $F$ be a field and $G \neq 1$ a countable locally finite group with no 
elements of order char $F$. If $G$ has no nonidentity finite normal subgroups, then $F[G]$ has a simple module which is not injective.

Proof. Suppose all simple $F[G]$-modules are injective. By [2], $F[G]$ is a primitive ring and hence, by Theorem $2, F[G]$ is a simple Artinian ring. By $[4$, p. 7], $G$ is finite and hence $G$ is a finite nonidentity normal subgroup contradicting the hypothesis.

\section{Group algebras.}

Lemma 8. Let $A$ be a locally Wedderburn algebra whose finite dimensional semisimple subalgebras can be chosen so that each is a direct sum of matrix algebras over (commutative) fields. If $V$ is a simple A-module finite dimensional over its commuting ring, then $\operatorname{Hom}_{A}(V, V)$ is a field.

Proof. It suffices to assume $A \simeq D_{n}$ for some division ring $D$ and prove that $D$ is a field. There is an idempotent $e \in A \ni e A e \simeq D$. If $x, y \in e A e$ choose a semisimple subalgebra $B$ containing $x, y$ and $e . e B e$ is the direct sum of algebras $f K_{m} f$ where $f$ is an idempotent in $K_{m}$ and $K$ is a field. However

$$
\exists r \leq m \quad \ni\left(\begin{array}{cc}
K_{r} & 0 \\
0 & 0
\end{array}\right) \simeq f K_{m} f \subseteq e A e
$$

has no zero divisors. Thus $f K_{m} f$ is isomorphic to $K$ or 0 and $e B e$ is commutative. In particular, $x$ and $y$ commute.

Lemma 9. (i) If char $F=p>0$ and $H$ is a finite group without p-elements then $F[H]$ is a sum of matrix algebras over fields.

(ii) If char $F=0, F$ has all roots of unity, and $H$ is a finite group, then $F[H]$ is a sum of matrix algebras over fields.

Proof. (i) By Maschke's theorem $F[G]$ is locally Wedderburn. Let $P \subseteq F$ be the prime field and let $H$ be a finite subgroup of $G . P[H]$ is a direct sum of matrix algebras over fields by Wedderburn's theorem on finite division algebras. Since no radical appears when tensoring up, the result follows.

(ii) This is an immediate consequence of the Brauer splitting theorem for finite groups [1].

Let $\Delta(G)=\{g \in G:[G: C(g)]<\infty\}$.

Lemma 10. Let $H=\Delta(H)$ be a linear group. Then $[H: Z(H)]<\infty$.

Proof. Choose $h_{1}, h_{2}, \cdots, h_{n} \in H$ to span the linear span of $H$ and set $Z=\bigcap_{i=1}^{n} C\left(h_{i}\right)$. Then $[H: Z]<\infty . Z$ centralizes the $h_{i}$ and hence their linear span. Thus $Z=Z(H)$.

Theorem 3. Let $F$ be either a field of char 0 with all roots of unity or a field of char $p>0$. Let $G$ be a countable group. Then all simple $F[G]-$ modules are injective if and only if $G$ is locally finite with no elements of order char $F$ and $G$ has an abelian subgroup of finite index. 
Proof. If $G$ has an abelian subgroup of finite index, then $F[G]$ has bounded representation degree [4] and so a simple $F[G]$-module is finite dimensional over its commuting ring. But $F[G]$ is locally Wedderburn and hence by Theorem 2 all simple $F[G]$-modules are injective.

Conversely, if all simple $F[G]$-modules are injective then, by Theorem $1, G$ is locally finite with no elements of order char $F$. We can write $G=\cup_{n=1}^{\infty} G_{n}$ where $G_{n} \subseteq G_{n+1}$ and each $G_{n}$ is finite. If there exist simple $F\left[G_{i}\right]$-modules $V_{i}$ and $F\left[G_{i}\right]$ monomorphisms $f_{i}: V_{i} \rightarrow V_{i+1}$ and if $\operatorname{dim}_{C_{i}} V_{i}$ is unbounded $\left(C_{i}\right.$ is the commuting ring of $\left.V_{i}\right)$, then as in Lemmas 5 and 6 , there exists a simple module infinite dimensional over its commuting ring. This contradicts Theorem 2 . Hence there must exist an $n$ and a simple $F\left[G_{n}\right]$-module $V$ such that if $m>n, W$ a simple $F\left[G_{m}\right]$ module and $f: V \rightarrow W$ a $F\left[G_{n}\right]$-monomorphism, then $\operatorname{dim}_{C(V)} V=\operatorname{dim}_{C(W)} W$.

Let $e$ be the central idempotent of $F\left[G_{n}\right]$ corresponding to $V$. Let $r=\operatorname{dim}_{C(V)} V$. Then $e F\left[G_{n}\right]=C(V)_{r}$ (the $r \times r$ matrix ring over $C(V)$ ). Let $m \geq n$. $F\left[G_{m}\right]$ $=S_{1} \oplus \cdots \oplus S_{t}$ where $S_{i}$ 's are minimal ideals. Write $e=a_{1}+\cdots+a_{t}$. If $a_{i} \neq 0$, then projection onto $S_{i}$ gives a monomorphism $C(V)_{r} \rightarrow S_{i}$ and hence there exists a $F\left[G_{n}\right]$-monomorphism from $V \rightarrow V_{i}$ where $V_{i}$ is a simple $S_{i}$-module. It follows that $S_{i}$ is also an $r \times r$ matrix ring and $a_{i}$ is the identity of $S_{i}$. Therefore $e$ is in the center of $F\left[G_{m}\right]$ and hence in the center of $F[G]$.

$e F[G]$ satisfies a polynomial identity. To see this let $W$ be a simple $e F[G]-$ module. Since $W e \neq 0$, there exists an $F\left[G_{n}\right]$-monomorphism $V \rightarrow W$. $\operatorname{dim}_{C(W)} W=r$; otherwise as in the proof of Lemma 6, there exists $G_{m}$ and $X$ a simple $F\left[G_{m}\right]$-module with $\operatorname{dim}_{C(X)} X>r$ and an $F\left[G_{n}\right]$-monomorphism $V \rightarrow X$, a contradiction. By Lemma $8, C(W)$ is a field whence $e F[G]$ is a subdirect sum of $r \times r$ matrix rings over fields. Thus $e F[G]$ satisfies the standard identity of degree $2 r$.

By Theorem 3.3 of [5], [G: $\Delta]<\infty$ and $\left|\Delta^{\prime}\right|<\infty$. For each $x \in \Delta^{\prime}$, not 1 , $1-x \notin J K[G]=0$; hence there exists an irreducible representation $\rho$ with $\rho(x) \neq 1$. If $\bar{\Delta}=\rho(\Delta)$ and $\bar{G}=\rho(G)$, then $\bar{G}$ is a linear group. By Lemma 10 , $[\bar{\Delta}: Z(\bar{\Delta})]<\infty$. Observe that $Z(\bar{\Delta}) \triangleleft \bar{G}$. If $A_{x}$ is the complete inverse image of $Z(\bar{\Delta})$ in $G$, then $A_{x} \subseteq \Delta,\left[\Delta: A_{x}\right]<\infty$, and $A_{x} \triangleleft G$. Since $\overline{A_{x}}$ is abelian and $x \neq 1$, it follows that $x \notin\left(A_{x}\right)^{\prime}$. Set $A=\cap\left\{A_{x}: x \in \Delta^{\prime}, x \neq 1\right\}$. Then $A \triangleleft G$ and $[G: A]<\infty$. $A$ is abelian since $A \subseteq \Delta$ and $A \cap \Delta^{\prime}=1$.

Remark. Alternatively, one may prove the last theorem by showing that all simple $K[G]$-modules are also finite dimensional over their commuting rings whenever $K$ is an algebraically closed field of char $F$. Standard techniques in finite group representation theory can be invoked to show that $C[G]$ shares the same property. Finally, one applies an analytic result of Thoma [6] to show that $G$ is abelian-by-finite.

\section{REFERENCES}

1. R. Brauer, On the representation of a group of order $g$ in the field of $g$-th roots of unity, Amer. J. Math. 67 (1945), 461-471. MR 7, 238.

2. E. Formanek and R. L. Snider, Primitive group rings, Proc. Amer. Math. Soc. 36 (1972), 357-360. 
3. G. O. Michler and O. E. Villamayor, On rings whose simple modules are injective, J. Algebra 25 (1973), 185-201.

4. D. S. Passman, Infinite group rings, Marcel Dekker, New York, 1971.

5.—, Group rings satisfying a polynomial identity. II, Pacific J. Math. 39 (1971), 425-438.

6. E. Thoma, Uber unitäre Darstellungen abzählbarer, discreter Gruppen, Math. Ann. 153 (1964), 111-138. MR 28 \#3332.

7. O. E. Villamayor, On weak dimension of algebras, Pacific J. Math. 9 (1959), 941-951. MR 21 \#7243.

DEPARTMENT OF MATHEMATICS, BRANDEIS UNIVERSITY, WALTHAM, MASSACHUSETTS 02154

DEPARTMENT OF MATHEMATICS, NORTHWESTERN UNIVERSITY, EVANSTON, ILLINOIS 60201 